\title{
Recusa vacinal - o ponto de vista ético
}

Paulo Santos,* Alberto Hespanhol*

\section{RESUMO}

A vacinação constitui uma das maiores vitórias da Medicina moderna, permitindo a prevenção de mais casos de doença e morte precoce do que qualquer outro tratamento médico. No entanto, o sucesso alcançado na diminuição do número de novos casos pode fazer esmorecer a motivação para o prosseguir, levando ao aparecimento de argumentos anti-vacinação que colhem em grupos mediaticamente muito ativos, e fazendo perigar a continuidade dos resultados alcançados.

Neste artigo são revistos os aspetos éticos relacionados com a questão da não adesão e recusa à vacinação, enquanto afirmação dos princípios éticos da liberdade e da autonomia, e reflete-se no papel dos profissionais de saúde e instituições nesta matéria.

Conclui-se que, mais importante do que a formalização de procedimentos para justificar a responsabilidade de quem recusa uma vacina, este momento deve ser encarado como uma oportunidade de fazer educação para a saúde, numa perspetiva de empoderamento do indivíduo e da população na lógica de uma melhor literacia que permita de facto a decisão informada, livre e esclarecida.

Palavras-chave: Ética Médica; Vacinação; Adesão ao Tratamento; Consentimento Informado; Recusa de Tratamento.

A vacinação define-se como o processo pelo qual a inoculação de um agente no corpo, seja um microrganismo ou uma substância, produz imunidade para uma determinada doença. ${ }^{1}$ A sua origem histórica perde-se nos primórdios do segundo milénio, com relatos chineses de inoculações variólicas para prevenção da varíola, o mesmo acontecendo em África e na Turquia, onde aliás teve origem a técnica que, em 1720, Lady Montagu introduziu em Inglaterra. ${ }^{2}$ Foi, no entanto, Edward Jenner quem trouxe uma abordagem de experimentação científica e em 1798 publicou os resultados da eficácia da inoculação do vírus da vaccinia (varíola bovina) na prevenção da varíola humana em 23 indivíduos, dando início à era moderna da vacinação. Demoraria quase um século para os trabalhos de Louis Pasteur trazerem algum incremento nesta área, com a descoberta da atenuação da virulência do agente, levando a uma explosão de conhecimento, traduzida pela descrição das vacinas contra a raiva e contra o antraz. A evolução ditou a erradicação da varíola, com o último caso descrito em 1977

* Médicos de família, Unidade de Medicina Geral e Familiar, Departamento de Ciências Sociais e Saúde, Faculdade de Medicina da Universidade do Porto. na Somália, e a quase erradicação da poliomielite prevista agora para 2020.

$\mathrm{Na}$ atualidade o número de vacinas é de vinte e cinco. ${ }^{3}$ A maioria dos países representados na Organização Mundial de Saúde (OMS) apresenta programas nacionais de vacinação estruturados, mais ou menos abrangentes, estimando-se uma redução mundial da mortalidade de cerca de 2,5 milhões de crianças por ano.

Em Portugal, a vacinação variólica inicia-se em 1894 e permanece obrigatória até 1977, e as vacinas do tétano e da difteria iniciaram-se com carácter obrigatório em 1962. O primeiro Programa Nacional de Vacinação foi publicado em 1965 e caracterizou-se pela distribuição universal e gratuita de vacinas à população, de acordo com um calendário definido e seguindo as orientações técnicas estabelecidas. Foi criado nesta altura o Boletim Individual de Saúde que faria prova da vacinação. A primeira vacina foi a da poliomielite, seguida em 1966 pelas do tétano, da difteria, da tosse convulsa e da varíola, notando-se nos anos seguintes uma notável redução da mortalidade e morbilidade pelas doenças infeciosas alvo de vacinação. ${ }^{4}$ Atualmente inclui recomendações para um conjunto de 12 vacinas estrategicamente distribuídas de forma a maximizar a proteção 
conferida na idade mais adequada e o mais precocemente possível.

Na avaliação de 2012 do Programa Nacional de Vacinação, promovida pela Direção Geral da Saúde (DGS), a taxa de cobertura na infância era superior a 95\%, mas a vacinação contra o tétano e difteria abrangia apenas $70 \%$ da coorte que completou 65 anos. ${ }^{5} \mathrm{~A}$ vacina contra as infeções pelo vírus do papiloma humano apresentava taxas de cobertura superiores a $85 \%$ para as 3 doses administradas em praticamente todas as coortes de avaliação. No entanto, reconhece-se que o enorme sucesso dos programas de vacinação pode levar à perceção de que o risco associado à vacina seja superior ao da própria doença, diminuindo a adesão e assim comprometer esse mesmo sucesso. ${ }^{6}$

O objetivo deste artigo é rever os aspetos éticos associados à opção de não adesão à vacinação pela população adulta e pelos pais/tutores da população infantil, contribuindo para uma reflexão sobre as atitudes e competências dos profissionais de saúde e das instituições nesta matéria.

\section{PRINCÍPIOS ÉTICOS DA VACINAÇÃO}

«Eu, solenemente, juro consagrar minha vida a serviço da Humanidade... Praticarei a minha profissão com consciência e dignidade... A saúde dos meus pacientes será a minha primeira preocupação». ${ }^{7}$

Este trecho, retirado do Juramento de Hipócrates conforme a revisão de 2006 da Declaração de Genebra da Associação Médica Mundial, situa a promoção da saúde como objetivo principal do exercício médico, numa atitude ética e deontológica direcionada para o paciente e orientada pelos valores do respeito pelo próximo, da beneficência, da autonomia, da não maleficência, da justiça e da verdade.

O respeito pelo paciente implica que o indivíduo tem o direito de ser tratado com dignidade e assume o acesso à melhor informação disponibilizada numa linguagem compreensível, garantindo a sua opção livre de escolher ou rejeitar o procedimento médico, que à partida é proposto a favor dos seus melhores interesses, numa ponderação que inclui o benefício esperado da intervenção, o seu risco e os seus custos.

No caso da vacinação, a prescrição de uma vacina, esteja ou não incluída num programa vacinal, é um ato médico que deriva de uma avaliação integrada onde se inclui a ponderação da sua eficácia e efetividade, da sua segurança, do seu impacto organizacional, do seu custo-benefício e das questões éticas, sociais e psicológicas associadas. ${ }^{8}$ Diversas agências em todo o mundo promovem ativamente esta avaliação e publicam recomendações onde disponibilizam a informação necessária para responder às questões levantadas. ${ }^{9-11} \mathrm{Em}$ Portugal a DGS é responsável pela publicação e atualização das normas de vacinação. ${ }^{4}$

Acresce nesta discussão que à avaliação individual se sobrepõe a questão do coletivo, que condiciona os valores da efetividade, introduzindo-se o conceito da imunidade de grupo em que o facto de uma proporção significativa de indivíduos estar imunizada para uma condição diminui o número de expostos em risco e, mantendo a base populacional, diminui consequentemente a incidência da doença. Esta é uma questão ética em que a autonomia do próprio está condicionada pela perspetiva comunitária do bem comum.

No caso dos menores, legalmente incapazes para consentir até aos 16 anos, os pais ou tutores assumem especial responsabilidade, tendo em atenção o dever de velar pela sua segurança e saúde, garantindo-lhes as oportunidades para um desenvolvimento saudável no limite do seu próprio potencial. Neste aspeto a autonomia dos pais ou tutores será indexada ao princípio da beneficência para a criança ou adolescente. A garantia do acesso à vacinação é um fator de equidade geracional e negar essa oportunidade pode equacionar a aptidão para o exercício da responsabilidade parental, sobretudo se culposamente colocar o menor numa situação de grave prejuízo.

\section{RECUSA DA VACINAÇÃO}

Nigenda-Lópezpublicou em 1997 uma revisão sobre os motivos para a não adesão à vacinação, ${ }^{12}$ categorizando-os por mitos culturais sobre a atuação médica, características socioeconómicas da população, falta de informação disponível, medo psicológico do próprio, dos pais ou dos profissionais de saúde e problemas organizacionais dos serviços de saúde. Estes fatores, apesar de alguma especificidade local, eram patentes em vários países analisados.

Os movimentos anti-vacinação são tão antigos quanto a própria vacinação e o próprio Jenner enfrentou a acusação de que tal ato seria contra a vontade divina. ${ }^{13}$ 
Um caso atual assume alguma relevância na Europa, nomeadamente na Holanda e na França, onde o benefício da profilaxia é contraposto ao potencial risco iatrogénico, numa sociedade em que a melhoria dos cuidados de higiene e a mudança de hábitos das popula- ções seriam fatores que por si só poderiam manter o padrão epidemiológico das doenças entretanto alcançado. ${ }^{14}$ Esta visão de tipo naturalista apresenta o problema de se basear em observações de doentes isolados ou de pequenas séries de casos, numa estrutura

\begin{tabular}{|c|c|c|c|c|c|c|c|c|c|c|c|c|c|c|}
\hline & $\mathbf{N}$ & BCG & HVB & VAT & VAD & VAPe & VIP & Hib & VAS & VAR & Parotidite & MenC-conj & HPV & Rotav \\
\hline Alemanha & 12 & & $x$ & $x$ & $x$ & $x$ & $x$ & $x$ & $x$ & $x$ & $x$ & & $x$ & \\
\hline Áustria & 12 & & $x$ & $x$ & $x$ & $x$ & $x$ & $x$ & $x$ & $x$ & $x$ & & $x$ & $x$ \\
\hline Bélgica & 13 & & $x$ & $x$ & $x$ & $x$ & $x$ & $x$ & $x$ & $x$ & $x$ & $x$ & $x$ & $x$ \\
\hline Bulgária & 13 & $x$ & $x$ & $x$ & $x$ & $x$ & $x$ & $x$ & $x$ & $x$ & $x$ & & & \\
\hline Chipre & 11 & & $x$ & $x$ & $x$ & $x$ & $x$ & $x$ & $x$ & $x$ & $x$ & & & \\
\hline Dinamarca & 11 & & $x$ & $x$ & $x$ & $x$ & $x$ & $x$ & $x$ & $x$ & $x$ & & $x$ & \\
\hline Eslováquia & 12 & $x$ & $x$ & $x$ & $x$ & $x$ & $x$ & $x$ & $x$ & $x$ & $x$ & & & \\
\hline Eslovénia & 12 & $x$ & $x$ & $x$ & $x$ & $x$ & $x$ & $x$ & $x$ & $x$ & $x$ & & $x$ & \\
\hline Espanha & 13 & & $x$ & $x$ & $x$ & $x$ & $x$ & $x$ & $x$ & $x$ & $x$ & $x$ & $x$ & \\
\hline Estónia & 10 & $x$ & $x$ & $x$ & $x$ & $x$ & $x$ & $x$ & $x$ & $x$ & $x$ & & & \\
\hline Finlândia & 14 & $x$ & $x$ & $x$ & $x$ & $x$ & $x$ & $x$ & $x$ & $x$ & $x$ & MenACWY & & $x$ \\
\hline França & 13 & $x$ & $x$ & $x$ & $x$ & $x$ & $x$ & $x$ & $x$ & $x$ & $x$ & $x$ & $x$ & \\
\hline Grécia & 16 & $x$ & $x$ & $x$ & $x$ & $x$ & $x$ & $x$ & $x$ & $x$ & $x$ & $x$ & $x$ & \\
\hline Hungria & 11 & $x$ & $x$ & $x$ & $x$ & $x$ & $x$ & $x$ & $x$ & $x$ & $x$ & & & \\
\hline Irlanda & 13 & $x$ & $x$ & $x$ & $x$ & $x$ & $x$ & $x$ & $x$ & $x$ & $x$ & $x$ & & \\
\hline Itália & 13 & $x$ & $x$ & $x$ & $x$ & $x$ & $x$ & $x$ & $x$ & $x$ & $x$ & $x$ & & \\
\hline Letónia & 14 & $x$ & $x$ & $x$ & $x$ & $x$ & $x$ & $x$ & $x$ & $x$ & $x$ & & $x$ & $x$ \\
\hline Lituânia & 11 & $x$ & $x$ & $x$ & $x$ & $x$ & $x$ & $x$ & $x$ & $x$ & $x$ & & & \\
\hline Luxemburgo & 15 & & $x$ & $x$ & $x$ & $x$ & $x$ & $x$ & $x$ & $x$ & $x$ & $x$ & $x$ & $x$ \\
\hline Malta & 12 & $\mathrm{x}$ & $x$ & $x$ & $x$ & $x$ & $x$ & $x$ & $x$ & $x$ & $x$ & & & \\
\hline Países Baixos & 12 & & $x$ & $x$ & $x$ & $x$ & $x$ & $x$ & $x$ & $x$ & $x$ & $x$ & $x$ & \\
\hline Polónia & 10 & $x$ & $x$ & $x$ & $x$ & $x$ & $x$ & $x$ & $x$ & $x$ & $x$ & & & \\
\hline Portugal & 13 & $x$ & $x$ & $x$ & $x$ & $x$ & $x$ & $x$ & $x$ & $x$ & $x$ & $x$ & $x$ & \\
\hline Reino Unido & 14 & & $x$ & $x$ & $x$ & $x$ & $x$ & $x$ & $x$ & $x$ & $x$ & $x$ & $x$ & \\
\hline República Checa & 11 & & $x$ & $x$ & $x$ & $x$ & $x$ & $x$ & $x$ & $x$ & $x$ & $x$ & & \\
\hline Roménia & 10 & $x$ & $x$ & $x$ & $x$ & $x$ & $x$ & $x$ & $x$ & $x$ & $x$ & & & \\
\hline Suécia & 12 & & $x$ & $x$ & $x$ & $x$ & $x$ & $x$ & $x$ & $x$ & $x$ & & $x$ & \\
\hline
\end{tabular}

BCG - vacina antituberculosa; VAT - vacina antitetânica; VAD - vacina antidiftérica; VAPe - vacina anti-tosse convulsa; VIP - vacina antipoliomielite inativada; Hib - vacina anti-Haemophilus influenzae tipo b; Parotidite - vacina anti-parotidite epidémica MenC-conj - Vacina antimeningococos do tipo C (conjugada); HPV - vacina anti-papilomavírus humano; Rotav - vacina anti-rotavírus; Pneumo-c - vacina antipneumocócica conjugada; TAB vacina anti-febre tifóide; VAG - vacina antigripal; HVA - vacina anti-hepatite A; HVB - Vacina anti-hepatite B; Raiva - vacina contra a raiva; Varicela vacina ati-varicela; $\mathrm{N}$ - número total de vacinas por país 
que, à luz das escalas de análise e decisão, se classifica como de baixa evidência. A sua divulgação maciça nos meios de comunicação e na internet, em linguagem que a maioria dos indivíduos consegue perceber, longe dos algoritmos matemáticos da publicação científi-

\begin{tabular}{c|c|c|c|c|c}
\hline & & & & \\
\hline
\end{tabular}

ca revista pelos pares, confere-lhe um poder efetivo de levar cada um, pelo menos, a questionar a certeza, por definição probabilística, da ciência médica, o que condiciona a adesão à vacinação. ${ }^{15} \mathrm{O}$ caso da possível associação da utilização do timerosal com o risco de vir a desenvolver autismo nas crianças é paradigmático, na medida em que nunca se conseguiu estabelecer o nexo de causalidade mas a polémica foi suficiente para determinar recusas à vacinação. ${ }^{16}$ Apesar da evidência científica publicada, a indústria farmacêutica fez um esforço para substituir o componente, restabelecendo a confiança. Neste momento não existem vacinas comercializadas em Portugal contendo timerosal.

A OMS publica normas para assegurar o controlo da segurança das vacinas, ${ }^{17-19}$ mas é fácil admitir que não existe nenhum procedimento que seja totalmente seguro. A maioria das reações às vacinas é ligeira e autolimitada como dor, rubor local e sintomas gerais de febrícula e mal-estar inespecífico, mas as raras reações graves como choque anafilático, alterações neurológicas e cegueira também estão descritas. Importa no entanto que estes efeitos sejam registados e alvo de avaliação contínua de farmacovigilância e que os resultados possam ser publicitados tanto nos meios profissionais como para a população, consubstanciando o princípio ético da verdade, correspondente à transmissão de informação ao doente de forma total, livre de preconceitos e de juízos de valor, e apontando os pontos positivos e os negativos, não esquecendo os eventuais riscos, de acordo com o estado da arte.

Em Portugal, o programa de vacinação proposto pela DGS inclui recomendações para um conjunto de 12 vacinas, a que acresce a vacinação contra a gripe, e não é muito diferente do que se propõe nos países da União Europeia (quadro I). O Despacho 17067/2011 do Gabinete do Secretário de Estado Adjunto do Ministro da Saúde, de 7 de dezembro de 2011, atualiza o Programa Nacional deVacinação, estabelece o esquema de vacinação universal para Portugal e fixa objetivos mínimos para uma cobertura nacional superior a 95\%. A DGS emitiu, em 21/12/2011, uma circular normativa onde estabelece a operacionalização do esquema proposto, e defende que, perante uma recusa, deverá o próprio ou o seu representante legal assinar uma declaração de recusa que ficará arquivada no serviço de vacinação, pressupondo essa possibilidade mas responsabilizando o seu autor na 
forma escrita. No entanto a regra geral do ordenamento jurídico português é a oralidade ${ }^{20} \mathrm{e}$ do ponto de vista formal o cidadão não está obrigado a assim proceder.

Apesar de alguma controvérsia ao longo dos anos, nunca foi entendido pelas sociedades ocidentais que a imposição das vacinas fosse uma intrusão demasiado profunda nos direitos e garantias dos cidadãos ou que manchasse o direito constitucional à liberdade. ${ }^{21} \mathrm{Em}$ Portugal, o esquema vacinal proposto poderá emanar do direito dos cidadãos à proteção da saúde e o seu cumprimento do dever de a defender e promover, conforme ao artigo $64 .{ }^{\circ}$ da Constituição da República. Não obstante, é uma proposta de cumprimento voluntário na medida em que apenas as vacinas antitetânica e antidiftérica são obrigatórias nos termos do Decreto-Lei n. ${ }^{\circ} 44.198$, de 20/02/1962, que nunca foi revogado. Encarar uma recusa à vacinação como uma oportunidade de fazer Educação para a Saúde e de poder moldar o preconceito à evidência do potencial benefício é uma atitude mais inclusiva ${ }^{22}$ que garantirá maiores ganhos em saúde do que claudicar no confronto, apondo um modelo de declaração para assinar.

\section{CONCLUSÃO}

A vacinação permite salvar mais vidas e prevenir mais casos de doença do que qualquer tratamento médico. ${ }^{4}$

É reconhecido ao indivíduo ou seu representante legal a possibilidade de consentir ou recusar qualquer procedimento médico, nomeadamente a vacinação, sendo que esse ato só poderá ser um exercício dos seus direitos de liberdade e de autonomia se realizado na posse da informação necessária. Prestar esta informação é dever dos serviços de saúde nos vários níveis da prestação de cuidados, institucional e pessoalmente, disponibilizando material informativo suficientemente inteligível, promovendo o empoderamento da população para uma melhor decisão em saúde, sendo também seu dever averiguar se o indivíduo percebeu a mensagem que foi transmitida.

É reconhecido ao indivíduo o dever de defender e promover a sua própria saúde e a dos outros, criando condições para a melhoria dos níveis de saúde da sociedade, nomeadamente através do contributo para o estabelecimento de uma imunidade de grupo que possa levar eventualmente à erradicação de outras doenças, como aconteceu no passado com a varíola.
Os indivíduos ou seus representantes legais podem recusar o ato de vacinação, bastando para tal uma declaração da sua vontade livremente assumida, uma vez que estejam na posse da totalidade da informação que necessitam para a decisão, o que deverá ficar registado no respetivo processo clínico para cada vacina excluída.

O respeito pelo princípio da autonomia não invalida um esforço acrescido de capacitação do indivíduo que recusa a vacina proposta no seu melhor interesse, reconhecendo que a decisão é revogável a todo o momento sem que tal possa implicar na qualidade assistencial que é prestada. A recusa das vacinas antidiftérica e antitetânica, que se mantêm obrigatórias para as crianças em Portugal, configura uma situação especial em que deve ser prestada informação sobre as consequências legalmente impostas em termos do acesso às atividades escolares e laborais. Nas crianças a recusa vacinal deve ser objeto de profunda reflexão pelos pais ou tutores numa análise cuidada do custo-benefício. Aos profissionais cabe respeitar a responsabilidade parental que, no entanto, se esgota no limite da avaliação da existência de perigo para a saúde da criança (artigos 1878. ${ }^{\circ}$ e $1918 .{ }^{\circ}$ do Código Civil). ${ }^{23}$

Em qualquer dos casos, a não adesão às vacinas é tão antiga como a própria vacinação e os profissionais envolvidos devem encarar cada acontecimento como uma oportunidade de fazer educação para a saúde, com o objetivo de aumentar a capacitação da população, contribuindo para a melhoria do seu nível de saúde.

\section{REFERÊNCIAS BIBLIOGRÁFICAS}

1. Feliciano J. A vacinação e a sua história. Cad DGS 2002 Out; 2: 3-7. Disponível em: http://www.dgs.pt/upload/membro.id/ficheiros/ i005533.pdf [acedido em 20/10/2012].

2. Plotkin SA. Vaccines: past, present and future. Nat Med 2005 Apr;11 (4 Suppl): S5-11.

3. WHO. Draft global vaccine action plan. Sixty-fifth World Health Assembly, 2012 - 11 May 2012. Report Nr:A65/22. Geneva:WHO; 2012.

4. Programa Nacional de Vacinação 2012, Norma DGS 040/2011. Lisboa: DGS; 2011.

5. DGS. Plano Nacional de Vacinação - Avaliação 2012. Lisboa: DGS; 2013.

6. DGS. Mitos sobre a vacinação. Lisboa: DGS; 2012.

7. World Medical Association. Declaration of Geneva. Disponível em: http://www.wma.net/en/30publications/10policies/g1/index.html [acedido em 20/10/2012].

8. Goodman C, National Information Center on Health Services Research \& Health Care Technology (U.S.). HTA 101 introduction to health technology assessment. Bethesda, MD: U.S. National Library of Medicine, National Institutes of Health, National Information Center on Health 
Services Research and Health Care Technology (NICHSR); 2004. Disponível em: http://www.nlm.nih.gov/nichsr/hta101/ta101_c1.html [acedido em 20/10/2012].

9. National Center for Immunization and Respiratory Diseases. General recommendations on immunization: recommendations of the Advisory Committee on Immunization Practices (ACIP). MMWR Recomm Rep 2011 Jan 28; 60 (2): 1-64.

10. National Advisory Committee on Immunization, Public Health Agency of Canada. Canadian Immunization Guide. 7th ed. Ottawa: Public Health Agency of Canada=Agence de la Santé Publique du Canada; 2006.

11. Salisbury D, Ramsay M, Noakes K, editors. Immunisation against infectious disease. 3rd ed. London: TSO; 2006.

12. Nigenda-López G, Orozco E, Leyva R. Motivos de no vacunacion: un analisis critico de la literatura internacional, 1950-1990. Rev Saude Publica 1997 Jun; 31 (3): 313-20..

13. Porter D, Porter R. The politics of prevention: anti-vaccinationism and public health in nineteenth-century England. Med Hist 1988 Jun; 32 (3): 231-52.

14. Smits T. Post-vaccination syndrome. Disponível em: http://www.postvaccination-syndrome.com [acedido em 20/10/2012]..

15. Nasir L. Reconnoitering the antivaccination web sites: news from the front.J Fam Pract 2000 Aug; 49 (8); 49(8):731-3.

16. National Advisory Committee on Immunization. Thimerosal: updated statement.An Advisory Committee Statement (ACS). Can Commun Dis Rep 2007 Jul 1; 33 (ACS-6):1-13.

17. Robertson J, Griffiths E.WHO guidelines for assuring the quality of DNA vaccines. Biologicals 1998; 26 (3): 205-12.

18. Arciniega JL, Corbel M, Dellepiane N, Dobbelaer R, Griffiths E, Heron I, et al. WHO guidelines for the production and control of the acellular pertussis component of monovalent or combined vaccines. Biologicals 1998 Sep; 26 (3): 195-204.

19. Corbel MJ, Das RG, Lei D, Xing DK, Horiuchi Y, Dobbelaer R, et al.WHO
Working Group on revision of the Manual of Laboratory Methods for Testing DTP Vaccines: Report of two meetings held on 20-21 July 2006 and 28-30 March 2007, Geneva, Switzerland. Vaccine 2008 Apr 7; 26 (16): 1913-21.

20. Oliveira G, Pereira AD. Consentimento Informado. Coimbra: Centro de Direito Biomédico; 2006.

21. Calandrillo SP.Vanishing vaccinations: why are so many Americans opting out of vaccinating their children? Univ Mich J Law Reform 2004 Winter; 37 (2): 353-440.

22. Schwartz JL, Caplan AL.Vaccination refusal: ethics, individual rights, and the common good. Prim care 2011 Dec; 38 (4): 717-28, ix.

23. Lei $n^{\circ}$. 23/2013, de 5 de Março de 2013. Diário da República 1. ${ }^{a}$ série - N. ${ }^{\circ} 45: 1220-35$.

\section{CONFLITO DE INTERESSES}

Os autores declaram não ter conflito de interesses.

\author{
ENDEREÇO PARA CORRESPONDÊNCIA \\ Paulo Santos \\ Unidade de Medicina Geral e Familiar \\ Departamento de Ciências Sociais e Saúde \\ Faculdade de Medicina da Universidade do Porto \\ Alameda Prof. Hernâni Monteiro \\ 4200-319 Porto \\ Portugal \\ e-mail: psantosdr@med.up.pt
}

Recebido em 03/08/2013

Aceite para publicação em 12/10/2013

Artigo escrito ao abrigo do novo acordo ortográfico.

\section{ABSTRACT}

\section{AN ETHICAL VIEW OF VACCINE REFUSAL}

Vaccination is one of the most important victories of modern medical science. It has prevented more cases of disease and premature death than any other medical treatment. But this success may decrease the motivation to continue with vaccination, resulting in the appearance of arguments against vaccination from groups with high media impact. This may endanger the outcomes achieved.

In this article, we review ethical aspects of poor adherence and vaccine refusal as expressions of patient autonomy and liberty. We reflect on the role of health professionals and institutions in addressing this question.

We conclude that an episode of vaccine refusal involves much more than the formal procedures undertaken to justify the responsibility of those making the decision. It is an opportunity for health education that may empower individuals and populations with better health literacy to allow truly free and informed decision-making.

Key-words: Medical Ethics; Vaccination; Patient Compliance; Informed Consent; Treatment Refusal. 